\title{
ENZYME-HISTOCHEMICAL STUDY ON THE NUCL. INTERPEDUNCULARIS OF THE RAT BRAIN*
}

\author{
KAtsuyuki IMAI \\ Department of Anatomy, School of Medicine, Akita University, Akita 010
}

Received for publication May 20, 1977, and in revised form June 13, 1977

\begin{abstract}
Histochemical studies have been conducted on the interpeduncular nucleus (IPN) of the rat. The neurons of such show a high level of hexokinase activity and are able to receive their energy supply directly from glucose in the circulating blood. These neurons are equally supplied with the enzymes of the Embden-Meyerhof pathway and with those of the hexose monophosphate shunt, indicating a high level of activity in the tricarboxylic acid cycle. Therefore, the rat IPN may belong to the category of "usual nuclei" $(15,16)$.

Neurons were classified into five categories based on various morphological patterns in the Golgi apparatus (GA). Type I and II neurons were dominant in the Corpus interpedunculare (Ci), and types III, IV and V neurons were dominant in the magnocellular nucleus (Mi) of the IPN. In general, the activities of the glycolytic enzymes such as hexokinase, aldolase, glyceroaldehyde-3-phosphate dehydrogenase, lactate dehydrogenase and succinate dehydrogenase were higher in the $\mathrm{Ci}$ than in the $\mathrm{Mi}$. These results indicate that the rat IPN may be classified histochemically into two subnuclei.

The high succinate dehydrogenase activity sporadically localized in the neuropil suggests that it may correspond to the structures formed by the horizontal axonplexus and by the richly ramified dendrites of the neurons in the IPN.
\end{abstract}

Since the first description of the interpeduncular nucleus (IPN) by Forel in 1872 (30), it has been described as an heterogeneous nucleus. In mammals such as the rabbit, rat, mountain beaver, guinea pig, chinchilla, capybara, cat, dog, mink, sheep, pig, monkey, and armadillo, the IPN may be divided into two to four subduclei $(2,19)$. A conclusion in regard to these subdivisions has yet to be clearly established, as seen in the disagreement between Ives (18) and Plante (30). Until recently, studies designed to determine the functional significance of the IPN have tended to assume that this structure may have homogeneous cytoarchitectonics $(21,28,44)$.

Enzyme-histochemical studies of the IPN were carried out on the rat, mouse, guinea pig, rabbit, cat, and squirrel monkey $(1,3-5,22,23,25,40)$. These reports indicated that succinate dehydrogenase $(\mathrm{SDH})$ and aldolase $(\mathrm{AD})$ reactions were strongly active, lactate dehydrogenase ( $\mathrm{LDH})$ moderately active, and glucose-6phosphate dehydrogenase (G6PD) weakly active in the IPN $(1,3-5,22,23,25$,

* Supported partly by grant-in aid for scientific research from the Ministry of Education, the Japanese Government (No. 177003). 
40). It is most important to note in these studies that the IPN is rich in both acethylcholine esterase and monoamine oxidase $(4,25)$. Recently, Manocha and Perachio (24) in studies on the effect of environmental light conditions, reported that the LDH reaction in both the neurons and neuropil of light-exposed squirrel monkeys showed a markedly increased enzyme activity in comparison to the control group of other animals. In spite of many histochemical reports on the IPN, no adequate description of the subunits of the IPN exits, as far as the author knows.

Iijima and Imai (16) have found three types of energy supply patterns to the neurons in the mesencephalon of rats. The first pattern may be observed in the Nucl. Edinger-Westphali (EW). These neurons derive their energy directly from glucose in the circulating blood, while inherent polysaccharide is not utilized as an energy source for these neurons. The second pattern may be observed in the Nucl. n. oculomotorii (III), the Nucl. ruber (R), and the pars lateralis of substantia nigra $(\mathrm{SN})$. These neurons may be able to derive their energy supplies from both the circulating blood and the surrounding glial cells. The third type of neuron is found in the pars compacta of SN, which derive energy only indirectly from the surrounding glial cells.

Friede (4) states that the locus ceruleus (LC), the nucleus dorsalis $n$. vagi (DVN), and the hypothalamic neurosecretory nuclei (HMN) may belong to the "exceptional nuclei". The neurons of these nuclei show an exceptional metabolic pattern which is rich in G6PD, LDH, NAD- and NADP-diaphorases, but low in $\mathrm{SDH}$ and cytochrome oxidase (CYO) and in enzymes involved in the tricarboxylic acid cycle (TCA-cycle). Iijima and Imai $(15,16)$ have carried out experiments on the conception of "exceptional nuclei" in regard to the glycolytic metabolism including hexokinase (HKase). They have studied the patterns of carbohydrate metabolism in the LC, DVN, III, SN, Nucleus tractus mesencephalicus n. trigemini, Nucleus $\mathrm{n}$. hypoglossi (XII), and $\mathrm{R}$ in rats. According to their report, the hexose monophosphate (HMP) shunt is predominant, rather than the Embden-Meyerhof (EM) pathway in the DVN, whereas an inverse relationship is found between the HMP and the EM in the LC: Therefore, this discrepancy strongly suggests that the LC is distinctly different from the DVN in regard to the pattern of carbohydrate metabolism, though both nuclei are included in the category of "exceptional nuclei". The MT and the XII are considered to be "usual nuclei" because the neurons in these nuclei derive energy directly from glucose in the circulating blood, and these two nuclei are equally supplied with both the EM pathway and with the HMP shunt, and are rich in enzymes involved in the TCA-cycle.

The thiamine pyrophosphatase (TPPase) method has been used to identify the Golgi apparatus (GA) in various regions of the central nervous system in the rat, rabbit, and chipmunk. The results, it is assumed, indicate cyclic changes of the $G A$ in the neurons studied $(7-16,35-38)$. With regard to the "exceptional nuclei" (11) of the rabbit and rat, studies have been published on the GA morphology and its possible role in the DVN, HMN, LC, SN and EW (15, 16). Iijima and Imai (15) have briefly described the morphological patterns of the GA in four nuclei of the rat mesencephalon, and Iijima (11) has made detailed studies on the histochemical patterns of the GA in the same nuclei (EW, III, R and $\mathrm{SN}$ ). He reports that on the basis of morphological patterns of the GA, the neuron in EW may belong to the "LC-DVN" type, and that the main type of the GA in most 
neurons of the III and in half neurons of the $\mathrm{R}$ may be motor in nature, whereas the other neurons in the III and R may belong to the category of "LC-DVN" type. The main type of GA in the $\mathrm{SN}$ neurons is much simpler than that of those described above.

In spite of the studies described above, little is known on the morphology of the GA and the pattern of carbohydrate metabolism in the IPN. In order to examine these questions, histochemical methods such as the TPPase method, HKase (EC. 2.7.1.1), $\alpha$-glucan phosphorylase (APase) (EC. 2.4.1.1), ADase (EC. 4.1.2.13), LDH (EC. 1.1.1.27 and 28), SDH (EC. 1.3.99.1), Glyceroaldehyde-3phosphate dehydrogenase (G3ase) (EC. 1.2.1.12), and G6PDase (EC. 1.1.1.49) methods as well as histological methods including HE-stain, rapid Golgi stain, Bielschowsky's impregnation method, and Cajal's gold sublimate method on the Nucleus interpeduncularis (IPN) of Wistar strain rats were used.

\section{MATERIALS AND METHODS}

Thirty three healthy adult male Wistar strain rats (body weight 200-350 g) were used in the present study. Immediately after the rats were killed by decapitation (without anesthesia) at 9 a.m., thin slices of the upper mesencephalon including the interpeduncular nucleus (IPN) were removed. The whole slices were quickly frozen in dry ice $\left(-55^{\circ} \mathrm{C}\right)$. Frozen slices were mounted on cryostat chucks, and $10 \mu \mathrm{m}$ cryostat sections were cut at $-20^{\circ} \mathrm{C}$ (Table 1 ).

In addition, Mayer's hematoxylin-eosin (HE) stain, rapid-Golgi stain, Bielschowsky's Silver impregnation, and Cajal's gold sublimate method were used on other slices of the mesencephalon. Sixty HE-stained frontal paraffin sections (five microns thick) from the three rats were measured for cell size with a micro-meter, and the mean was calculated with $\overline{\mathrm{X}}=\frac{1}{\mathrm{n}} \sum_{\mathrm{i}=1}^{\mathrm{n}} \mathbf{x}_{\mathbf{i}}$.

\section{RESULTS}

\section{(A) Histological results}

According to current results with the $\mathrm{HE}$ stain and the Bielschowsky's silver

\begin{tabular}{ll}
\multicolumn{1}{c}{ Enzymes } & TABLE 1 \\
\hline $\begin{array}{l}\text { 1. Hexokinase } \\
\text { 2. } \alpha \text {-glucan phosphorylase } \\
\text { 3. Glucose-6-phosphate } \\
\text { dehydrogenase }\end{array}$ & $\mathrm{HK}$ \\
$\begin{array}{l}\text { 4. Aldolase } \quad \mathrm{G} 6 \mathrm{PD} \\
\text { 5. Glyceroaldehyde-3- } \\
\text { phosphate dehydrogenase }\end{array}$ & $\mathrm{AD}$ \\
$\begin{array}{l}\text { 6. Lactate dehydrogenase } \\
\text { 7. Succinate dehydrogenase } \\
\text { 8. Thiamine pyrophosphatase }\end{array}$ & $\mathrm{G} 3$ \\
\hline
\end{tabular}

Methods Used

Meijer (1967)

Takeuchi \& Kuriaki (1955)

Barka \& Anderson (1963)

Abe \& Shimizu (1964)

Himmelhoch \& Karnovsky (1961)

Barka \& Anderson (1963)

Nachlas et al. (1957)

Novikoff \& Goldfischer (1961) 
impregnation method, the rat IPN is subdivided into two parts; the corpus interpedunculare $(\mathrm{Ci})$ and the magnocellular nucleus of the IPN (Mi). The arrangement of these submuclei is shown schematically in Fig. 19. The Mi lies on the anterior border of the $\mathrm{Ci}$ and extends to the caudal border of the mamillary bodies (Fig. 19).

\section{(1) Mayer's hematoxylin-eosin stain}

The diameters of the nerve cell bodies and their nuclei were measured in the

$\mathrm{Ci}$ (Fig. 1) and in the Mi by Plante's (29) method. In the $\mathrm{Ci}$, the mean diameter of nerve cell bodies was $10.6 \pm 1.3 \mu$ and the mean diameter of their nuclei was 7.6 $\pm 1.3 \mu$. In the mi, the mean diameter of nerve cell bodies was $12.7 \pm 2.3 \mu$ and that of their nuclei was $9.3 \pm 1.7 \mu$.

(2) Bielschowsky's silver impregnation method

The IPN was in sharp contrast to the darkly stained surrounding tissue. In frontal sections, many transversal and perpendicular fibers were seen in the IPN (Fig. 2). The density of transversal fibers was higher in the $\mathrm{Ci}$ than in the $\mathrm{Mi}$ (Fig. 2). There was little difference between the $\mathrm{Ci}$ and the $\mathrm{Mi}$ in regard to the density of perpendicular fibers. Therefore, total fiber density was much greater in the $\mathrm{Ci}$ than in the $\mathrm{Mi}$. However, a direct connection between these fibers and neurons in the IPN was not discerned.

\section{(3) The rapid Golgi method}

The IPN was sharply contrasted to the darkly stained surrounding area. In the $\mathrm{Ci}$, the neuron was of a multipolar type and its dendrites had numerous spines. In the Mi, the neuron was of a multipolar type, but its dendrites were shorter and dendritic spines fewer than those observed in the $\mathrm{Ci}$. The rapid Golgi method revealed a large number of transversal fibers in the $\mathrm{Ci}$ (Fig. 3). In the IPN, these fibers had many short collateralis, and many fibers terminated as a tufty ramification (Fig. 4). Other fibers made terminals with some short secondary branches seen in empty space. The empty space appeared to correspond to a nerve cell body (Fig. 4).

\section{(4) Cajal's gold sublimate method}

Many astrocytes were seen in the $\mathrm{Ci}$ and $\mathrm{Mi}$. Some astrocytes showed glia pedicles and other astrocytes surrounded the nerve cell bodies with their processes. However, no neuron surrounded by a considerable number of astrocytes was observed. There was no difference between the $\mathrm{Ci}$ and the $\mathrm{Mi}$ in regard to the density of the distribution of astrocytes.

\section{(B) Histochemical results (Table 2)}

(1) Hexokinase (EC 2.7.1.1) (HKase)

In reaction at a low magnification (Fig. 5), the Mi was mildly positive, whereas the $\mathrm{Ci}$ was strongly positive. In the $\mathrm{Ci}$, the perikarya of neurons and glial cells were strongly positive, but the neuropil in the $\mathrm{Ci}$ showed only a moderately positive reaction (Fig. 6). In the $\mathrm{Mi}$, the perikarya of neurons and glial cells were moderately positive and the neuropil was mildly positive. Contacts between neurons and glial cells were often observed in the $\mathrm{Ci}$ and the $\mathrm{Mi}$, although $\mathrm{HK}$ positive glial cells showed no tendency to surround a neuron in either of the subnuclei. 
TABLE 2 Histochemical reactions in the rat IPN

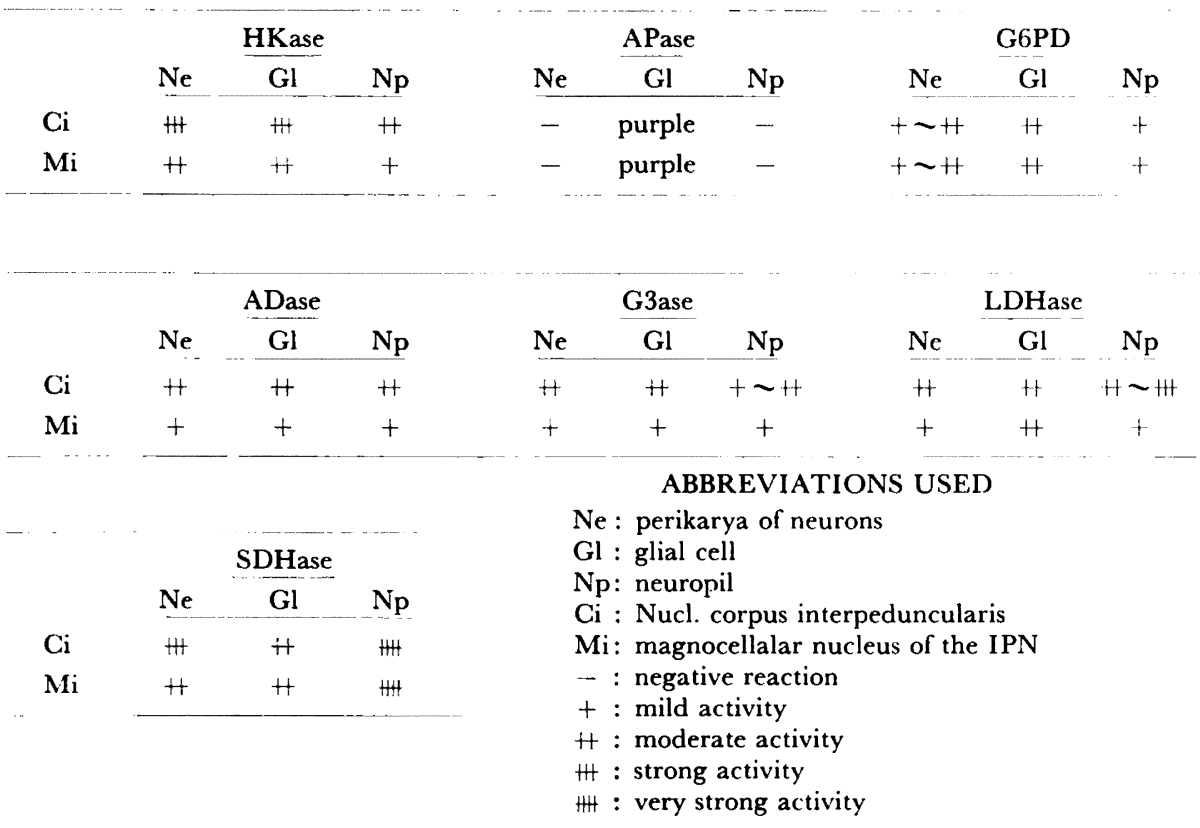

(2) a-glucan phosphorylase (EC 2.4.1.1) (APase)

In both $\mathrm{Ci}$ and $\mathrm{Mi}$ subnuclei, the cytoplasm and nuclei of the neurons showed a negative reaction to the APase test (Fig. 7). The glial cells exhibited purple granules in their perikarya, but the processes of glial cells were devoid of a reaction. The neuropil was also negative in the test (Fig. 7). APase-positive glial cells were distributed almost equally in the $\mathrm{Ci}$ and $\mathrm{Mi}$ in regard to density. APase-positive glial cells exhibited no tendency to surround the neurons.

(3) Glucose-6-phosphate dehydrogenase (EC 1.1.1.49) (G6PD)

In the G6PD reaction, no difference was noted between the $\mathrm{Ci}$ and $\mathrm{Mi}$. Both subnuclei showed a moderately positive reaction to the test at a low magnification. The perikarya of neurons showed a moderately to mildly positive reaction (Fig. 8). In the IPN, most glial cells showed moderate activity and other glial cells were weakly positive. The neuropil of both subnuclei was mildly positive.

(4) Aldolase (EC 4.1.2.13) (ADase)

In most cases, glial cells did not contact the neurons and were randomly distributed. The Mi showed mild activity and the Ci exhibited moderate activity at a low magnification. The perikarya of neurons, glial cells and the neuropil showed moderately positive activity in the Ci (Fig. 9), whereas they showed mild activity in the Mi.

(5) Glyceroaldehyde-3-phosphate dehydrogenase (EC 1.2.1.12) (G3ase)

The reaction in the $\mathrm{Mi}$ was mildly positive and in the $\mathrm{Ci}$ was moderately positive. In the $\mathrm{Ci}$, the perikarya of neuron and glial cells showed moderate activity and the neuropil exhibited mild to moderate positive activity (Fig. 10). 
In the $\mathrm{Mi}$, the perikarya of neurons, glial cells and the neuropil showed a mildly positive reaction. However, the intensity of the reaction in the neuropil of the Ci varied. The neuropil of the $\mathrm{Ci}$ had moderately positive flecks (Fig. 10, arrows $=$ F) which were dispersed and irregularly shaped. These moderately positive flecks were randomly scattered in the mildly positive background and often had cores which revealed strongly positive granules. Also, the neuropil of the Mi had a tendency to react unevenly, as seen in the $\mathrm{Ci}$. In the Mi however, the flecked positive areas were indistinct because of their mildly positive reaction. Neurons and glial cells were randomly distributed.

(6) Lactate dehydrogenase (EC 1.1.1.27. and 28.) ( $L D H)$

The reaction in the $\mathrm{Mi}$ was mildly positive, whereas the $\mathrm{Ci}$ was moderately positive. In the $\mathrm{Ci}$, the perikarya of the neurons and glial cells were moderately positive, and the neuropil was unevenly moderate to strongly positive for the LDH test (Fig. 11). The strongly positive areas in the neuropil of the $\mathrm{Ci}$ contained more strongly positive aggregations of granules (Fig. 11). On the other hand, the unevenness of the reactions in the $\mathrm{Ci}$ were observed in the neuropil of the $\mathrm{Mi}$ as well, but the reactions in the $\mathrm{Mi}$ were mildly positive, thus weaker than in the $\mathrm{Ci}$. The neurons were not surrounded by glial cells. Although a close relationship between neurons and glial cells was observed in a few cases, most glial cells were sporadically distributed.

\section{(7) Succinate dehydrogenase (EC 1.3.99.1) $(S D H)$}

Among all the enzyme-histochemical tests used in this study this nucleus reacted most intensely to the SDH reaction test. The Mi was moderately positive while the $\mathrm{Ci}$ was very strongly positive (Fig. 12). In the $\mathrm{Ci}$, the perikarya of the neurons showed a strong reaction (Fig. 12) while the glial cells were moderately positive and the neuropil showed the strongest positive reaction (Fig. 12). In the $\mathrm{Mi}$, the perikarya of the neurons and glial cells showed a moderately positive

FIG. 1. HE stain in the Mi. The cell body of this neuron is 13 microns in the mean diameter and its nucleus is 9 microns in the mean diameter. This nucleus shows three infoldings in the nuclear membrane (arrows). $\times 1,500$

Fig. 2. Bielschowsky's silver impregnation in the IPN. The interpeduncular nucleus of the rat has numerous transversal fibers. However, density of the fibers is different in the $\mathrm{Mi}$ and $\mathrm{Ci}$. It is higher in the $\mathrm{Ci}$ (lower half part, letter $\mathrm{Ci}$ ) than in the $\mathrm{Mi}$ (upper half part, letter $\mathrm{Mi}$ ). $\quad \times 60$

FIG. 3. Rapid Golgi method preparation of the $\mathrm{Ci}$ (low magnification). Note the presence of large numbers of fibers in this subnucleus and the fibers having many short and some long branches (arrows). $\quad \times 300$

Fig. 4. Rapid Golgi method preparation of the Ci (high magnification). The transversal fibers and their branches terminate into tufty ramifications (arrows). The empty spaces correspond to the nerve cell bodies $(\mathrm{Ne}) . \quad \times 1,500$

FIg. 5. The interpeduncular nucleus showing the HKase reaction (low magnification). Note the difference in reaction between $\mathrm{Mi}$ and $\mathrm{Ci}$. The $\mathrm{Mi}$ (upper half part, letter $\mathrm{Mi}$ ) is mildly positive, whereas the $\mathrm{Ci}$ (lower half part, letter $\mathrm{Ci}$ ) is strongly positive.

The glial cells (arrows $\mathrm{G}$ ) and nerve cell bodies (arrows $\mathrm{Ne}$ ) showed strong activity in the $\mathrm{Ci}$ and moderate activity in the $\mathrm{Mi} . \quad \because 300$

Fig. 6. The Ci showing HKase reaction (high magnification). Although the nuclei (arrows $\mathrm{N}$ ) show a negative reaction, the cytoplasm of these neurons (arrows $\mathrm{Cy}$ ) exhibits strong positive reaction. The neuropil $(\mathrm{Np})$ of the $\mathrm{Ci}$ shows a moderate positive reaction. $\quad \times 1,500$ 


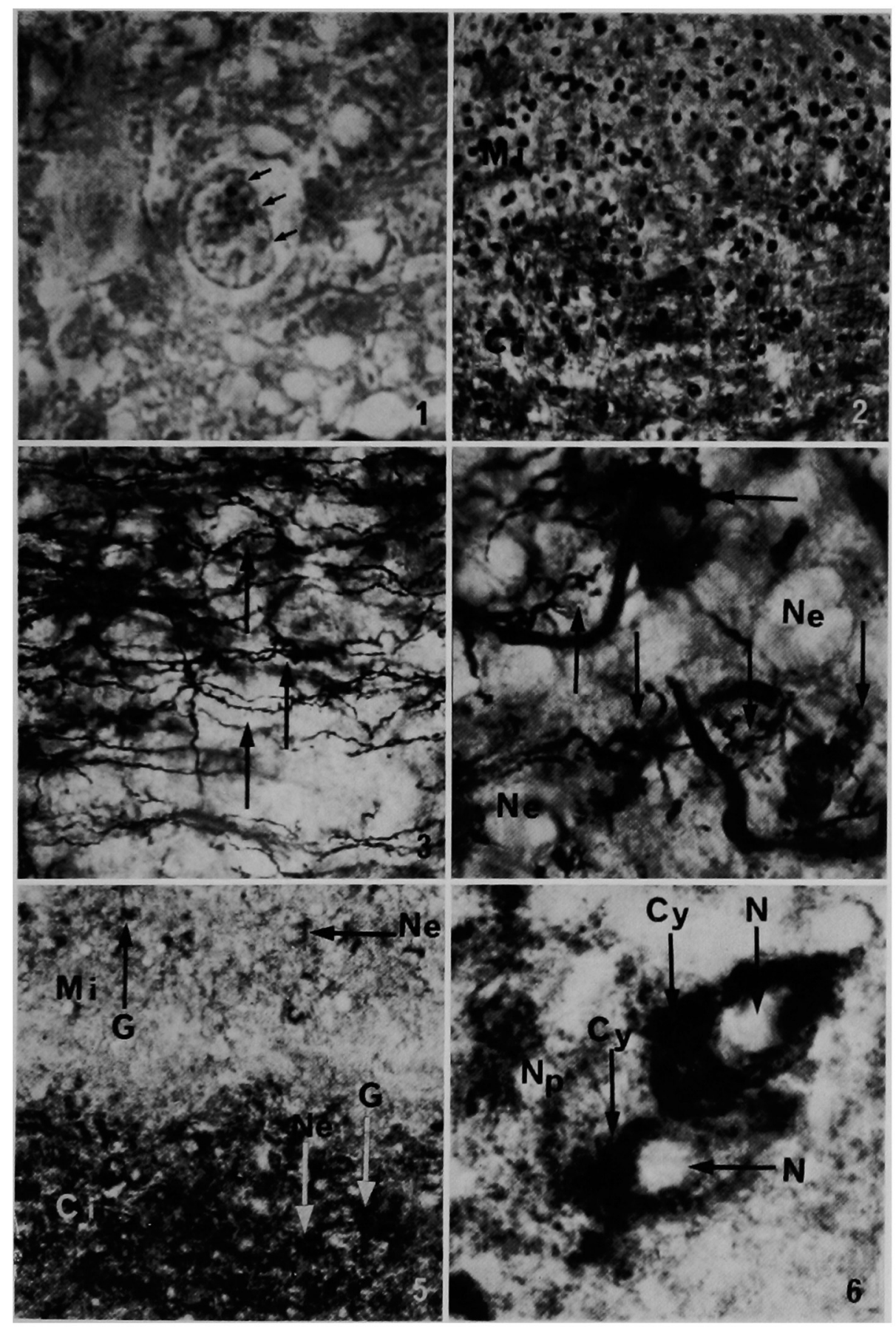




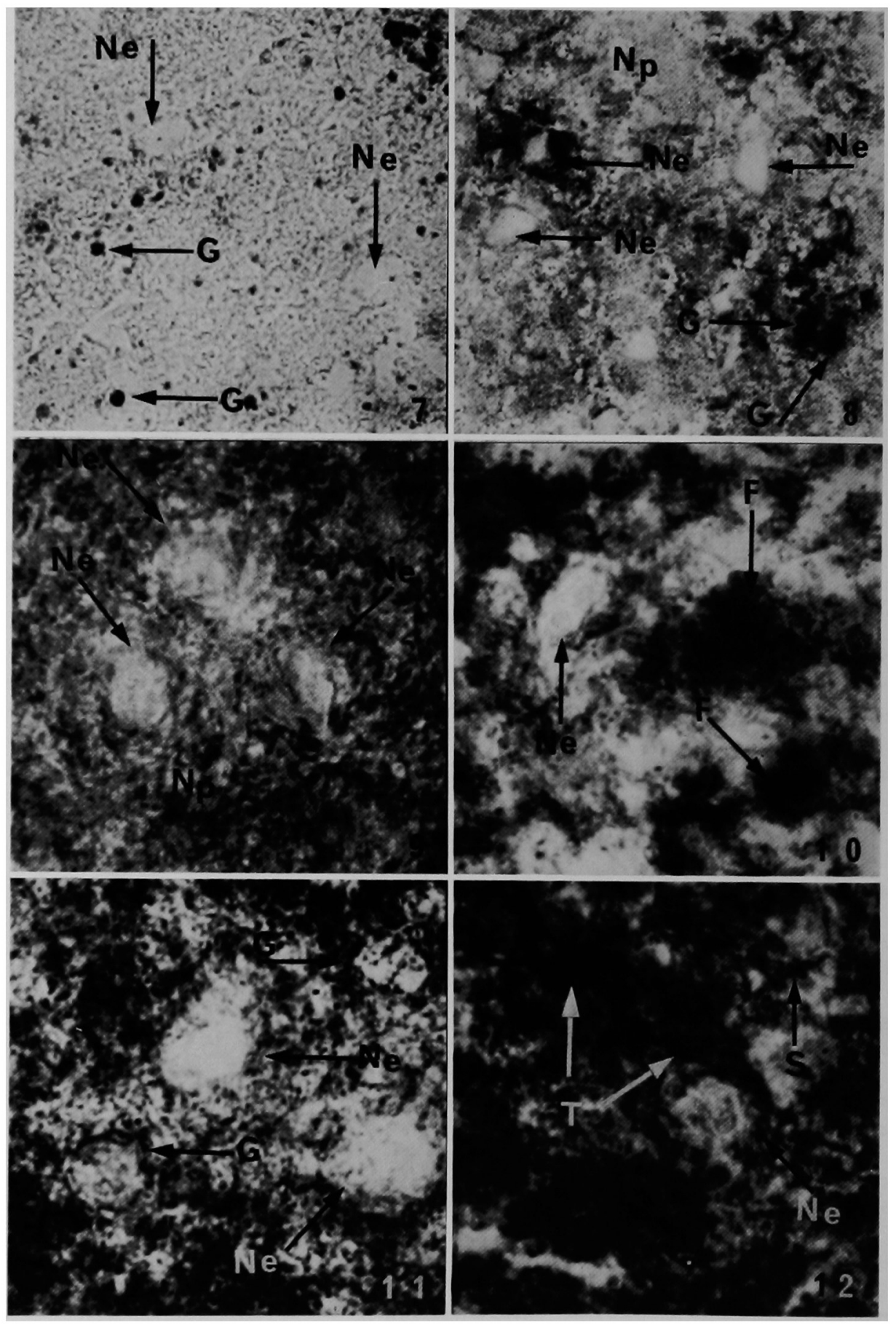


reaction. The variable reactions observed in the neuropil were seen more clearly in the $\mathrm{SDH}$ reaction than in the $\mathrm{AD}, \mathrm{G} 3$, and $\mathrm{LDH}$ reactions. The neuropil of the $\mathrm{Ci}$ contained many strongly positive areas (Fig. 12). They resembled the terminals of transversal fibers observed in rapid Golgi-stained specimens. In the $\mathrm{Mi}$, like structures of those described above were also observed, but they reacted more mildly than those in the $\mathrm{Ci}$. In both subnuclei, the glial cells contacted neurons in only a few cases. No reaction was observed in the neurons surrounded by numerous glial cells.

\section{(8) Thiamine pyrophosphatase (TPPase)}

A single neuron showed different types of TPPase reactions (Figs. 13-18). Its nucleus and nucleolus showed a negative reaction. TPPase-positive material (GA) was observed in all nerve cell bodies which occasionally extended into their dendrites. Occasionally, two or three such extensions ran in different directions. The neurons in the IPN were classified into five categories based on the morphological patterns of the GA, according to the classification introduced by Iijima (7-9) (Fig. 14-18).

\section{Type I cells.}

In this group, the GA was composed of granules and vesicles evenly distributed in the perikaryon (Fig. 14). Short strands connected to the granules and vesicles showed poor development.

2. Type II cells.

In this group, the GA consisted of granules, vesicles and thin strands interconnecting these elements (Fig. 15). The fine strands often developed into localized reticular networks. Although the localized reticular networks were restricted to a portion of the perikaryon or were randomly distributed, as a whole they did not constitute a complete reticular network.

3. Type III cells.

In this group, the GA consisted of perinuclear strands connected to many granules and small masses. These strands inconsistantly surrounded the nucleus

Fig. 7. The Ci showing APase reaction. The glial cells (arrows G) are stained purple, while the cytoplasm and nuclei of the neurons (arrows $\mathrm{Ne}$ ) are negative. $\times 600$

Fic. 8. The $\mathrm{Ci}$ showing G6PD reaction. The perikarya of the neurons (arrows $\mathrm{Ne}$ ) show a mildly to moderately positive reaction. The glial cells (arrows $G$ ) show a strongly positive reaction. The neuropil $(\mathrm{Np})$ shows a mildly positive activity. $\times 600$

FIG. 9. The $\mathrm{Ci}$ showing ADase reaction. The perikarya of neurons (arrows $\mathrm{Ne}$ ) and the neuropil (Np) show moderately positive activity. $\times 1,500$

FIG. 10. The Ci showing G3ase reaction. The perikarya of neurons (arrow Ne) show moderately positive activity. Note the presence of moderately positive flecks (arrows F) in the mildly positive neuropil. $\times 1,500$

Fig. 11. The Ci showing $\mathrm{LDH}$ reaction. The perikarya of neurons (arrows $\mathrm{Ne}$ ) and glial cells (arrows G) are moderately positive and the neuropil is moderately to strongly positive for the reaction. $\times 1,500$

Fig. 12. The $\mathrm{Ci}$ showing $\mathrm{SDH}$ reaction. The cytoplasm of a neuron (arrow $\mathrm{Ne}$ ) shows a strong reaction. Note the presence of the fine strand-like structure (arrow $\mathrm{S}$ ) and the strongly positive tufty areas (arrows $T$ ) in the strongly positive neuropil. $\times 1,500$ 


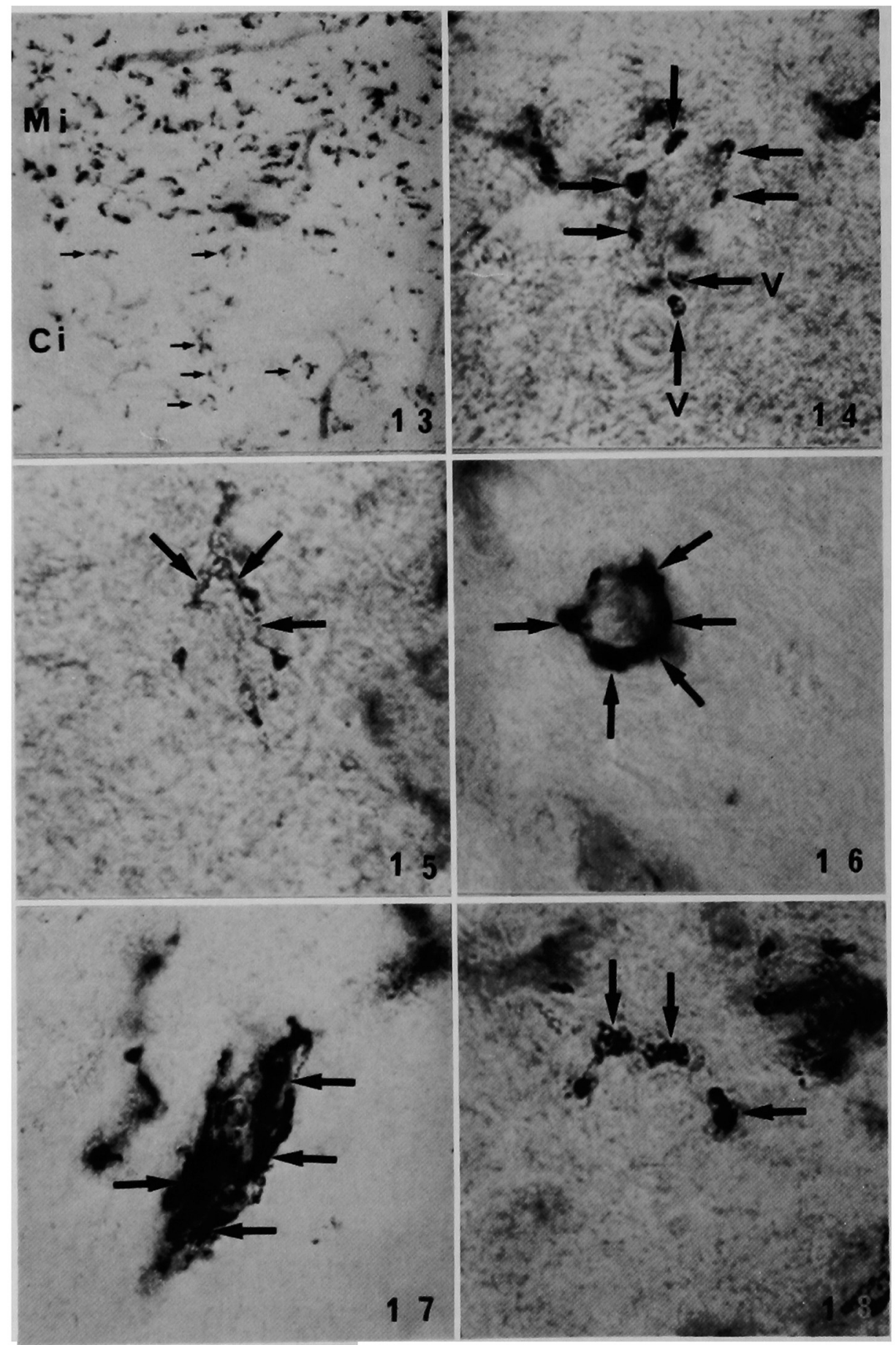




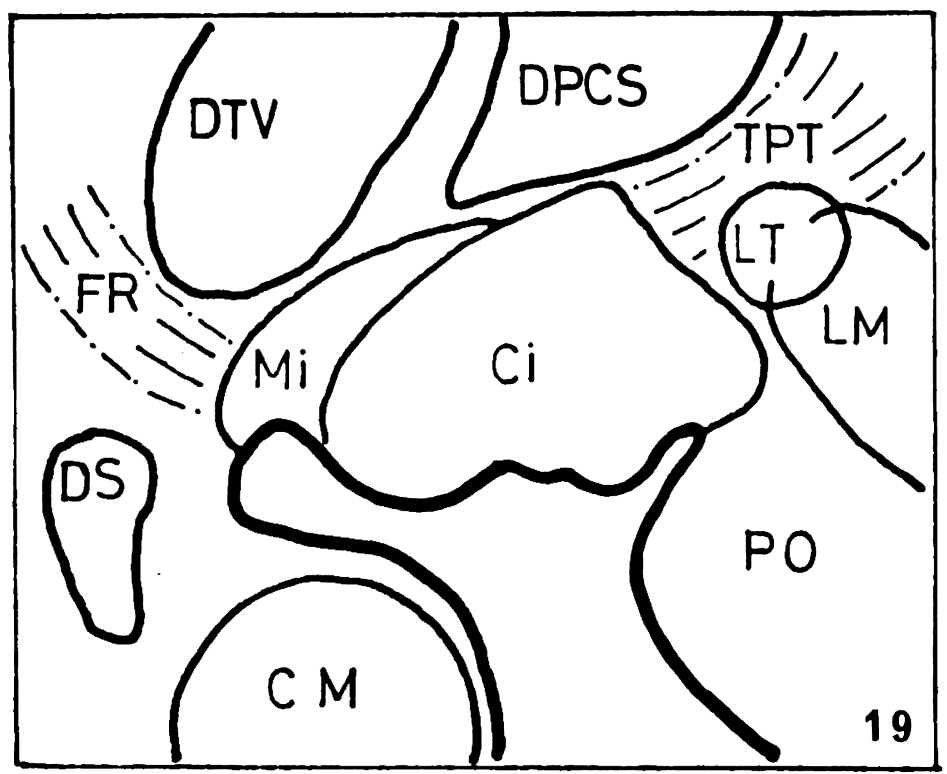

Fig. 19. Semidiagramatic illustration of the subnuclei of the IPN in the rat brain, as shown in the medial sagittal plane. The magnocellular nucleus (Mi) of the IPN is situated between the rostral top of the corpus interpedunculare ( $\mathrm{Ci}$ ) and the most caudal point of the corpus mamillare (CM). With the exception of the $\mathrm{Ci}$ and the Mi, these areas were identified according to the atlas of the rat brain written by Konig and Klippel (21). Abbreviations used in this figure: DTV, Decussatio tegmenti ventralis; DPCS, Decussatio pedunculorum cerebellarium superiorum; TPT, Tractus pedunculotegmentalis; FR, Fasciculus retroflexus; Mi, Magnocellular nucleus; Ci, Corpus interpeduncular nucleus; LT, Lemniscus medialis; DS, Decussatio supramamillaris; PO, Pons. $\quad .40$

Fig. 13. TPPase preparation of the interpeduncular nucleus of a rat at a low magnification. In comparing the $\mathrm{Ci}$ (lower half part, letter $\mathrm{Ci}$ ) with the $\mathrm{Mi}$ (upper half part, letter $\mathrm{Mi}$ ), a difference is seen in regard to the degree of positive activity. The individual neuron of the $\mathrm{Ci}$ (small arrows) shows a simpler Golgi apparatus than that in the $\mathrm{Mi} . \quad \times 90$

Fic. 14. The Ci showing TPPase reaction. The Golgi apparatus of this type I neuron is composed of evenly distributed granules (arrows) and vesicles (arrows V). $\quad \times 1,500$

FIG. 15. The C.i showing TPPase reaction. The Golgi apparatus of this type II neuron consists of granules and thin strands (arrows) interconnecting these granules incompletely. $\times 1,500$

Fig. 16. The Mi showing TPPase reaction. The Golgi apparatus of this type III neuron consists of perinuclear strands connected to many coarse granules (arrows). $\quad \times 1,500$

FIG. 17. The Mi showing TPPase reaction. The Golgi apparatus of this type IV neuron is composed of a reticular network (arrows) with many strongly positive masses. The network occupied the major part of the perikaryon. $\times 1,500$

Fig. 18. The Mi showing TPPase reaction. The Golgi apparatus of this type V neuron is composed of separate localized reticular networks (arrows) connected to many fine granules. $\quad \times 1,500$ 
(Fig. 16). Some neurons had the secondary branches originating from the main strands.

4. Type IV cells.

In this group, the fundamental morphological pattern of the GA was a reticular network which occupied the greater part of the perikaryon (Fig. 17). This reticular network included masses, many granules and vesicles.

5. Type $V$ cells.

In this group, the GA was composed of a few masses or separate localized reticular networks in the perikaryon (Fig. 18). The strands interconnecting these elements were less developed than those observed in the other types.

As described above, the neurons in the IPN were classified into five categories, but there were some neurons whose GA indicated an overlapping of various categories. In comparing the $\mathrm{Ci}$ with the $\mathrm{Mi}$, Types I and II were predominant in the Ci, while Types III, IV and V were predominant in the Mi (Fig. 13). The GA of the neurons in the $\mathrm{Ci}$ tended to be simpler in structure, with fewer granules, masses and strands than that of the neurons in the Mi. In particular, an individual neuron of Type III or IV in the Mi contained many more masses than that in the Ci. This difference was clearly observable at a low magnification as well. (Fig. 13).

\section{DISCUSSION}

Initially, patterns of energy supply to neurons in the rat IPN will be discussed in relation to the present results of HKase and APase reactions. It has been biochemically confirmed that the brain tissue receives its energy supply mainly from glucose in the circulating blood $(27,45)$. Some histochemical studies have confirmed that the glial cells act as an energy donor to the nerve cells in various nuclei of the brain, including Nucl. Deiters of the cat, bulbus olfactorius and the cerebellum of the squirrel monkey, DVN, and XII of the rabbit, and LC and DVN of the rat $(6,8,15,34)$. Most importantly, in their histochemical study on the rat mesencephalon, Iijima and Imai (16) classified the patterns of energy supply to neurons into three categories, based on the patterns of $\mathrm{HK}$ and APase reactions. The $\mathrm{Ci}$ in the IPN of the rat showed strong activity in the nerve cell perikarya and moderately positive activity in glial cells and the neuropil for the HKase test, but did not show APase activity in nerve cell bodies for the present study (41, 43). In the $\mathrm{Mi}$, the perikarya of nerve cells and glial cells showed moderate positive HKase activity, and only the glial cells showed a purple coloration for the APase reaction indicating that the glial cells may have an ability to synthesize or break down glycogen. However, since the IPN of the rat does not contain glycogen after the fourth week of birth (31), the glial cells do not utilize the inherent polysaccarides. The present results in regard to $\mathrm{HK}$ ase and APase reactions indicate that the pattern of energy supply to neurons in the rat IPN may belong to the second category of Iijima and Imai's classification (16). It seems most likely that nerve cells get their energy supplies from both the surrounding glial cells and glucose in the circulating blood (16). In the present study, this consideration is supported by the presence of many glia pedicles and by the processes of astrocytes surrounding the nerve cell bodies in the specimens of Cajal's gold sublimate stain. Also, in the rat $\mathrm{Mi}$, the 
nerve cells derive energy supplies in both above-mentioned ways, because the nerve cells were moderately positive and the neuropil was mildly positive for the HKase reaction. There is however, a difference between the $\mathrm{Ci}$ and the $\mathrm{Mi}$ in the quantity of glucose intake.

The distribution of enzymes involved in the course of glycolysis has been reported in relation to the rat, guinea pig, rabbit, mouse and squirrel monkey $(8,11,39$, 60 ). According to these studies, the IPN was moderately positive for the G6PD and $\mathrm{LDH}$ reactions, and strongly positive for the $\mathrm{AD}$ and $\mathrm{SDH}$ reactions $(3,5,25$, 40). In comparing these prior results with the present, with the exception of the $\mathrm{AD}$ reaction which was moderately positive in the present study, they are consistent with each other in regard to the $\mathrm{Ci}$ of the rat. It seems most likely that previous authors neglected the subdivisions of the IPN. Friede (5) observed in the SDH reaction of the guinea pig IPN that the "dorsal portion" showed a slightly weaker reaction than the "ventral portion", and that there was a difference between these two subdivisions. However, he did not record the difference between the small neuron and the neuropil in the $\mathrm{Ci}$ for the $\mathrm{SDH}$ reaction. In this respect, the present results indicate that the neurons are strongly positive, whereas the neuropil is very strongly positive for SDH reaction in the rat IPN. In the neuropil, axon terminals or synapses-like structures revealed greater SDH activity than the other structures. In the $\mathrm{Mi}$, the neurons are moderately positive and the neuropil is strongly positive in reaction, this is different than that of $\mathrm{Ci}$.

The carbohydrate metabolism in the IPN will be discussed below. Friede (4) described the DVN, LC, HMN, EW and the pars compacta of the SN as belonging to "exceptional nuclei" because of their exceptional metabolic pattern of nerve cell bodies. Recently, Iijima and Imai (15) have made particular note of the fact that glial cells surround neurons and that they may serve as energy donors to neurons in the LC and DVN of the rat. They also note that the EM pathway may be dominant in the LC, whereas the HMP shunt is dominant in the DVN, and that both the MT and XII-which belong to the "usual nuclei"-are equipped equally with the HMP shunt and with the EM pathway. They have presented a new definition in regard to the "usual nuclei", namely that neurons in the "usual nuclei" show a strong HKase activity and have the ability to derive their energy supply directly from glucose in the circulating blood, that the neurons are equipped equally with the enzymes of the EM pathway and with the HMP shunt, and that they show a high level of activity in the TCA cycle. According to their new criterion, III, XII, and R of the rat should belong to "usual nuclei". On the basis of the present results concerning HKase, G6PD, AD and SDH, the $\mathrm{Ci}$ of the IPN in the rat should also belong to the "usual nuclei". Although the levels of the enzyme activities of the EM pathway and of the TCA cycle in the Mi were slightly lower than those in the $\mathrm{Ci}$ in the present study, the neurons in the $\mathrm{Mi}$ can be considered to belong to the "usual nuclei" also.

The GA in the neurons of the rat IPN was divided into five different categories in the present study, in which many intermediate forms could be observed. These findings indicate that the GA in the neurons of this nucleus undergo cyclic changes $(7-16,32,33,35-38)$. The neurons in the Mi contained well developed GA, but they showed moderately positive HKase activity, which may indicate that the neuron does not receive large amounts of energy supply. It is noteworthy that the 
GA did not show the supranuclear position nor the bunches of grapes (9). However, there are clear differences between $\mathrm{Ci}$ and $\mathrm{Mi}$ of the rat IPN in regard to the types of the GA of the neurons. Types I and II were predominant in the $\mathrm{Ci}$, while Types III, IV and V were predominant in the Mi. Therefore, the rat IPN was clearly subdivided into two parts with the types of the GA of the neurons included. As to why the GA of the neurons in the Mi are better developed than those in the $\mathrm{Ci}$, it may simply be because the nerve cell bodies in the $\mathrm{Mi}$ are larger than those in the $\mathrm{Ci}$, as the large pyramidal cell has a well developed GA compared with that of a small pyramidal cell in the cerebral cortex of the rat (37). There is however, a significant possibility that the neurons in the Mi may participate in the active production of any synthesized material.

In the present study, the EM pathway and TCA cycle in the neurons of the $\mathrm{Ci}$, whose GA was poorly developed, showed greater activity than those in the $\mathrm{Mi}$. The reason for such may be the former supply of rich energy to the synapses in the neuropil through their dendrites, and the greater density of the synpases in the $\mathrm{Ci}$ than in the Mi. Because the neuropil itself showed a high HKase activity, there is a possibility that the synapses themselves may utilize glucose. Type I and II cells were dominant in the $\mathrm{Ci}$, whereas type III, IV and $\mathrm{V}$ cells were dominant in the Mi for the TPPase reaction in this study. This difference indicates that the subunits of the IPN may be composed of different neurons or the identical neurons in different functional states (8). It seems reasonable to conclude that the $\mathrm{Ci}$ and $\mathrm{Mi}$ of the rat IPN may consist of different cell groups respectively, for all the neurons in the $\mathrm{Ci}$ showed higher levels of activity in HKase, AD, G3, LDH and SDH tests than those in the Mi.

High levels of enzyme activity involved in the EM pathway as well as in the TC.A cycle, and the variability of their distribution in the Ci may be due to the presence of many axonal terminals in the neuropil of the Ci (4). Synaptic glomeruli are present in the rat IPN (21). These structures consist of multiple axonal endings of the horizontal axon's plexus which are engulfed three-dimentionally by sheetlike dendritic processes of the neuron in the IPN, and are up to 20 microns in diameter (21). Thus, it seems that there are numerous special synapses in the neuropil of the rat IPN, including the synaptic glomeruli.

On the other hand, there have been many enzyme-histochemical studies on the glomeruli in the cerebellum and in the olfactory bulb $(3,4,12,17,26,34,39$, $46,47)$. The granular layer of the cerebellum showed strongly positive oxidative enzyme activities which gave a "flocculated" appearance to this layer. These areas are considered to correspond to the cerebellar glomeruli $(3,4,34,46,47)$. Furthermore, Iijima (12) recently observed round or band-like positive areas and many fine granules in cerebellar glomeruli by applying G6PD, AD, G3, LDH and SDH reactions to the rat cerebellum, and he concluded that these structures may represent cross or longitudinal sections of a mossy fiber and aggregations of reaction products at the sites of mitochondria in synapses within the glomerulus (12). The glomeruli of the olfactory bulb also reveal high oxidative enzyme reactions $(4,17,26,29)$. The strong SDH activity localized in the neuropil is considered to be due to the fact that the neuropil has many synaptic terminals with many mitochondria. In the neuropil of the $\mathrm{Ci}$, there are a large number of synaptic terminals as observed in the sections stained by the rapid Golgi method, and various types of synapses such as 
the crest type and glomerulus type synapses which were revealed by electron microscopic study (21). Therefore, it seems reasonable to conclude that the spotted "flocculated" appearance of distribution of strong SDH activity in the rat IPN may be due to the presence of a high concentration of numerous synaptic terminals.

The rat IPN showed flecked strong activity in the neuropil for the G3, LDH and SDH reactions, but did not appear as such in the G6PD and AD reactions. The reason for differences of reaction between the neuropil of the IPN and cerebellar glomeruli may be due to the aggregations of synapses in the IPN which are not sufficiently developed so as to be equipped with complete glycolytic pathways in the cerebellar glomeruli (12).

\section{ACKNOWLEDGEMENT}

I wish to express my sincere gratitude to Prof. Dr. Koichi Iijima, head of the Anatomical department, Akita University School of Medicine, for his direction and encouragement, and to Mr. Naosuke Kojima for his technical assistance.

\section{REFERENCES}

1. Abe, T.. Yamada, Y., Hashimoto, H. and Shimizu, N.: Histochemical study of glucose-6phosphate dehydrogenase in the brain of normal adult rat. Med. J. Osaka Univ. 14; 69, 1963.

2. Cajal, R. S.: Histologie du Systeme Nerveux de L'homme et des Vertebres. Consejo superior de investigaciones cientificas, Madrid, 1972, p. 272.

3. Friede, R. L.: Histochemical investigations on succinic dehydrogenase in the central nervous system, III. Atlas of the midbrain of the guinea pig, including pons and cerebellum. J. Neurochem. 4; 290, 1959.

4. Friede, R. L.: Topographic Brain Chemistry. Accademic Press, New York, 1966, p. 74.

5. Friede, R. L. and Fleming, L.: A mapping of the distribution of lactic dehydrogenase in the brain of the rhesus monkey. Amer. J. Anat. 113;215, 1963.

6. Hydén, H. and Pigon, A.: A cytophysiological study of the functional relationship between oligodendroglial cells and nerve cells of Deiters nucleus. J. Neurochem. 6; 57, 1960.

7. Iijima, K.: Histochemical studies on the morphology of the Golgi apparatus and on the relationship between the Golgi apparatus and lysosomes in the cellular elements of the area postrema with the thiamine pyrophosphatase and acid phosphatase methods. Acta Histochem. (Jena) 33; $101,1969 \mathrm{a}$.

8. Iijima, K.: Histochemical studies on the morphology of the Golgi apparatus and on the distribution of hexokinase, phosphoglucomutase and some dehydrogenases in the dorsal vagal and hypoglossal nuclei of the rabbit. Histochemie $18 ; 132,1969 \mathrm{~b}$.

9. Iijima, K.: Histochemical studies on the morphology of the Golgi apparatus in the neurons of supraoptic and paraventricular nuclei of the normal and dehydrated rabbit. (application of the thiamine pyrophosphatase method). Z. Zellforsch. 103; 460, 1970.

10. Iijima, K.: Histochemical studies on the morphology of the Golgi apparatus on the distribution of hexokinase, L-gulonolactone oxidase, xylulose reductases, and ascorbic acid in the locus coeruleus of the rabbit. Histochemie 25; 107, 1971.

11. Iijima, K.: On the morphology of the Golgi apparatus in various nuclei of the rat mesencephalon (application of the thiamine pyrophosphatase method). Histochemistry 46; 61, 1975.

12. Iijima, K.: Histochemical studies on the morphology of the Golgi apparatus and on the distribution of some enzymes concerned with carbohydrate metabolism in the rat cerebcllum. Acta Histochem. (Jena) 58; 163, 1977. 
13. Iijima, K. and Awazi, N.: Histochemical studies on the morphology of the Golgi apparatus and its relationship to catecholamine biosynthesis in the locus coeruleus of the rat. Z. Zellforsch. $136 ; 329,1973$.

14. Iijima, K. and Imai, K.: Histochemical studies on the morphology of the Golgi apparatus and on the distribution of hexikinase, amylophosphorylase, cholinesterase and monoamine oxidase in the area postrema of the Asiatic Chipmunk. Acta Histochem. (Jena) 50; 163, 1974.

15. Iijima, K. and Imai, K.: Histochemical studies on the distribution of some enzymes concerned with carbohydrate metabolism in locus coeruleus, nucl. tractus mesencephalicus n. trigemini, nucleus dorsalis n. vagi and nucleus n. hypoglossi of the rat. Acta Histochem. (Jena) 52; 145, 1975.

16. Iijima, K. and Imai, K. Histochemical studies on the morphology of the Golgi apparatus and on the enzymes of carbohydrate metabolism in various nuclei of the rat mesencephalon. Histochemistry 46; 209, 1976.

17. Iijima, K., Shantha, T. R. and Bourne, G. H.: Histochemical studies on the distribution of seme enzymes of the glycolytic pathways in the olfactory bulb of the squirrel monkey. Histochemie $10 ; 224,1967$.

18. Ives, W'. R.: The interpeduncular nuclear complex of selected rodents. J. Comp. Neurol. 141; 77. 1971.

19. Kappers, C. U. A., Huber, G. C. and Crosby, E. C.: The Comparative Anatomy of the Nervous System of V'ertebrates, including Man. Hafner, New York, 1967, p. 1083.

20. König, J. and Klippel, R.: The Rat Brain. Krieger, New York, 1967.

21. Lenn, N. J.: Synapses in the interpeduncular nucleus, electron microscopy of normal and habenula lesioned rats. J. Comp. Neurol. 166; 73, 1975.

22. Manocha, S. L. and Bourne, G. H.: Histochemical mapping of Monoamine oxidase and lactic dehydrogenase in the pons and mesencephalon of squirrel monkey. J. Neurochem. 13; 1047, 1966.

23. Manocha, S. L. and Bourne, G. H.: Histochemical mapping of succinic dehydrogenase and cytochrome oxidase in the pons and mesencephalon of squirrel monkey (Saimiri sciureus). Exp. Brain Res. 2; 230, 1966.

24. Manocha, S. L. and Perachio, A. A.: Histochemical correlates of changes in the environmental light conditions. Hislochemie 34; 217, 1973.

25. Manocha, S. L. and Shantha, T. R.: Enzyme Histochemistry of the Nervous System. vol. 3., in The structure and Function of Nervous Tissue. ed. by Bourne, G. H., Academic Press. New York, 1969, p. 137.

26. Matsuo, S.: Histochemical studies on carbohydrate metabolism in the olfactory bulb of the rabbit. Bull. Tokyo Med. Dent. Univ. 15; 197, 1968.

27. McIlwain, H. and Bachelard, H. S.: Biochemistry and the Central Nervous System. Churchill Livingstone, London, 1971, p. 11.

28. Mizuno, N. and Nakamura, Y.: An electron microscope study of terminal degeneration of the fasciculus retroflexus mynerti within the interpeduncular nucleus of the rabbit. Brain Res. 65; $165,1974$.

29. Ogawa, K., Takeuchi, T. and Mori, T.: New Histochemistry. (in Japanese), Asakura, Tokyo, 1975.

30. Plante, S.: The comparative anatomy of the interpeduncular nucleus in the brain of the rat, cat and monkey. J. Neurol. Sci. 16; 155, 1972.

31. Sato, S.: Postnatal changes of glycogen in the brain of rats. Osaka Univ. Med. J. 11; 869, 1969.

32. Shantha, T.R. and Bourne, G. H.: The thiamine pyrophosphatase technique as an indicator of the morphology of the Golgi apparatus in the neurons. V, Studies on sympathetic ganglion cells. Cytologia (Tokyo), 31; 132, 1966.

33. Shantha, T. R. and Bourne, G. H.: The thiamine pyrophosphatase technique as an indicator of the morphology of the Golgi apparatus in the neurons. VI, Studies on the spinal cord, hippocampus and trigeminal ganglion. Ann. Histochem. 11;337, 1966.

34. Shantha, T. R., Iijima, K. and Bourne, G. H.: Histochemical studies on the cerebellum of 
squirrel monkey (Saimiri sciureus). Acta Histochem. (Jena) 27; 129, 1967.

35. Shanthaveerappa, T. R. and Bourne, G. H.: Histochemical demonstration of thiamine pyrophosphatase and acid phosphatase in the Golgi region of the cells in the eye. J. Anat. 99; 103, 1965.

36. Shnthaveerappa, T. R. and Bourne, G. H.: The thiamine pyrophosphatase technique as an indicator of the morphology of the Golgi apparatus in the neurons. I. Studies on ganglion cells. Acta Histochem. (Jena) 22; 155, 1965.

37. Shanthaveerappa, T. R. and Bourne, G. H.: The thiamine pyrophosphatase technique as an indicator of the morphology of the Golgi apparatus in the neurons. II. Studies on the cerebral cortex. Cellule $65 ; 201,1965$.

38. Shanthaveerappa, T. R. and Bourne, G. H.: The thiamine pyrophosphatase technique as an indicator of the morphology of the Golgi apparatus in the neurons. III. Studies on the olfactory bulb. Exp. Cell Res. 40; 292, 1965.

39. Shanthaveerappa. T. R. and Bourne, G. H.: Histochemical studies on the olfactory glomeruli of the squirrel monkey. Histochemie 5; 125, 1965.

40. Shimizu, N. and Abe, T.: Histochemical studies of the brain with reference to glucose metabolism. Prog. Brain Res. 21 ; 197, 1966.

41. Shimizu, N. and Kumamoto, T.: Histochemical studies on the glycogen of the mammalian brain. Anat, Rec. 114; 479, 1952.

42. Shimizu, N. and Morikawa, N.: Histochemical studies of succinic dehydrogenase of the Brain of mice, rats, guinea pig and rabbits. J. Histochem. Cytochem. 5; 334, 1957.

43. Shimizu, N. and Okada, M.: Histochemical distribution of phosphorylase in rodent brain from newborn adults. J. Histochem. Cytochem. 5; 459, 1957.

44. Smaha, L. A. and Kaelber, W. W: Efferent fiber projections of the habenula and interpeduncular nucleus. An experimental study in the opossum and cat. Exp. Brain Res. 16; 291, 1973.

45. Takagaki, G.: Metabolism of the cerebral tissue. In Biochemistry of the Brain, ed. by Tsukada, Y. Igaku Shoin Ltd. Tokyo, 1964, p. 93.

46. Tewari, H. B. and Bourne, G. H.: Histochemical distribution of enzymes associated with the metabolism of carbohydrates in the various elements of the cerebellum. Exp. Cell Res. 28; 444, 1962.

47. Tewari, H. B. and Bourne, G. H.: Histochemical studies on the distribution of oxidative enzymes in the cerebellum of the rat. J. Histochem. Cytochem. 10;619, 1962. 